\title{
Aplicación de inductores generadores de valor para la gestión en microempresas
}

\author{
Inductors generators application of value management in small enterprises
}

Recibido: 11-03-2016 • Aprobado: 12-06-2016 • Página inicial: 99 - Página final: 116

\author{
Jorge Iván Jiménez Sánchez* \\ Farley Sary Rojas Restrepo**
}

Resumen: este artículo propone la aplicación de inductores de valor que apoyen la gestión en las microempresas, pues a diferencia del análisis financiero tradicional, los inductores sirven para apreciar el progreso del dinamismo empresarial. Este método de análisis permite obtener información clave de los estados financieros para el cálculo de los inductores EBITDA, Margen EBITDA, El indicador KTNO capital de trabajo neto operativo, el indicador PKT (productividad de capital de trabajo), los indicadores de rentabilidad ROÍ, ROA, ROE, La PDC (palanca de crecimiento) y ver sus efectos. En este caso se aplicó un diagnóstico basado en inductores de valor a una empresa; los resultados se cotejaron con la dinámica del sector y se encontró que la empresa no genera valor para sus propietarios, presenta una rentabilidad inferior al compararla con otras empresas similares, pues el análisis de los inductores muestra que existe un riesgo financiero alto.

Palabras clave: inductores de valor, rentabilidad, crecimiento, valor, microempresas.

\begin{abstract}
This paper proposes the application of value drivers that support microbusiness management. Unlike traditional financial analyses, value drivers can be used to see the progress of entrepreneurial dynamism. This analysis method offers the possibility to obtain key information about financial statements in order to calculate and see the effects of EBITDA (Earnings before Interest, Taxes, Depreciation and Amortization), EBITDA margin, NOWC (Net Operating Working Capital), working capital productivity, ROI (Return on Investment), ROA (Return on Assets), ROE (Return on Equity) and growth levers. In this case, a diagnosis based on value drivers was applied to a company, and the results were compared with the sector's dynamics. It was found that the company does not generate value for its owners, and has lower profitability compared with other similar companies, since the analysis of drivers shows that there is a high financial risk.
\end{abstract}

Keywords: Value drivers, profitability, growth, value, small businesses.

* MSc. en Administración, docente de tiepo completo e integrante del Grupo de Investigación GORAS de la Facultad de Ciencias Administrativas, Económicas y Contables de la Fundación Universitaria Luis Amigó, Medellín - Colombia. jijs294@gmail.com

** MSc. en Administración, docente de tiepo completo e integrante del Grupo de Investigación CONTAS de la Facultad de Ciencias Administrativas, Económicas y Contables de la Fundación Universitaria Luis Amigó, Medellín - Colombia. frojasrestrepo@yahoo.com 


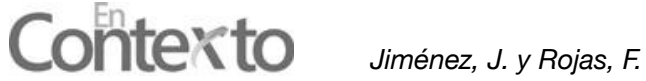

\section{Mise en oeuvre des inducteurs générateurs de valeur pour la gestion des microentreprises}

Résumé: cet article propose la mise en oeuvre des inducteurs de valeur qui soutiennent la gestion des micro-entreprises, car contrairement à l'analyse financière traditionnelle, les inducteurs servent à apprécier le progrès du dynamisme des entreprises. Cette méthode d'analyse permet d'obtenir les informations essentielles des états financiers pour le calcul des inducteurs EBITDA, KTNO, PKT (productivité du capital de travail), les indicateurs de rentabilité ROÍ, ROA, ROE, la PDC (levier de croissance) et de voir leurs effets. Dans ce cas a été appliqué un diagnostic basé sur des inducteurs de valeur à une entreprise; les résultats ont été comparés avec la dynamique du secteur et on a constaté que l'entreprise ne génère pas de valeur pour ses propriétaires, présente une rentabilité inférieure à comparer avec d'autres entreprises similaires, car l'analyse des inducteurs montre qu'il existe un risque financier élevé.

Mots-clés: inducteurs de valeur, rentabilité, croissance, la valeur, microentreprise.

\section{Implementação de geradores de indutores de valor para a gestão de microempresas}

Resumo: este artigo propõe a aplicação de indutores de valor que suportam o gerenciamento em microempresas, porque ao contrário da análise financeira tradicional, indutores servem para avaliar o progresso do dinamismo empresarial. Este método de análise permite a obtenção de informações chave nas demonstrações financeiras para o cálculo do EBITDA margem EBITDA, o indicador de KTNO capital de giro líquido, o indicador PKT (produtividade do capital de giro), os indicadores de rendibilidade ROÍ, ROA, ROE, o PDC (alavanca de crescimento) e ver os seus efeitos. Neste caso aplicado um diagnóstico baseado em indutores de valor para uma empresa; os resultados foram comparados com a dinâmica do sector e que se verificou que a empresa não gerar valor para os seus proprietários, apresenta uma rentabilidade inferior quando comparado com outras empresas similares, desde a análise da indutores mostra que existe um risco financeiro alto.

Palavras-chave: valor indutores,rentabilidade, crescimento, o valor, microempresa. 


\section{Introducción}

El artículo se fundamenta en la aplicación de inductores generadores de valor enfocado a la gestión en microempresas, pero ¿qué es un inductor de valor? Un inductor de valor es un indicador que muestra los componentes de la operación del negocio que por tener relación causa-efecto unos con otros, muestran en su resultado el por qué de su aumento o disminución en las cifras que son producto de las decisiones tomadas (García, 2003, p.177).

El profesor García expone estos indicadores financieros y los llama inductores de valor, también explica la manera correcta de interpretar sus resultados. Para este trabajo investigativo toma algunos de los más importantes como El EBITDA, el Margen EBITDA, PKT, la palanca de crecimiento y el cómputo del KTNO (capital de trabajo neto operativo). Todos los inductores anteriores son la base para calcular el inductor palanca de crecimiento (PDC), y encierran una importancia central porque "inducen" a los gerentes a trazar las metas y objetivos, y su resultado enfoca la gerencia en el concepto de generación de valor (García, 2003, p.486).

El diagnóstico financiero tradicional no está dirigido a la creación de valor, porque no aporta plena información y se fundamenta en datos pasados. La microempresa de hoy requiere aplicar modelos de diagnósticos basados en inductores de valor. Con el fin de analizar su resultado, se propone aplicar este método a una microempresa que fue tomada al azar entre una base de datos de 169 empresas del sector que cumplen la condición para ser microempresas de acuerdo con la Ley 590 de 2000 y la Ley 905 de 2004 (Congreso de la República de Colombia, 2010).

Se seleccionó para tal fin la empresa Industrias Tago S. A., que pertenece a la división productos textiles, prendas de vestir y calzado. Para el análisis se tomaron los estados financieros de los años 2008-2013 y se extrajeron las cifras necesarias para la aplicación de los inductores de valor. Se aprecia como la compañía genera utilidades pero no rentabilidad, sus indicadores ROA, ROE, ROÍ así lo muestran, pués cambian abruptamente, pasando de un crecimiento a la destrucción de valor. La empresa tiene problemas de liquidez, que el ciclo de efectivo refleja, porque un peso que sale de la empresa tarda cuarenta días en retornar nuevamente. Industrias Tago S. A. y las empresas del sector no tienen un crecimiento positivo. Al asociar el Margen EBITDA y la PKT se obtiene la PDC (palanca de crecimiento), este nuevo índice muestra qué tan atractivo es para una firma su crecimiento y representa si este contribuye a mejorar el valor a los propietarios; en este caso se tuvo un resultado inferior a uno. 


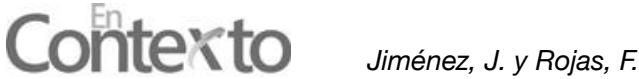

\section{Metodología}

Al igual que García (2003), se propone una metodología para realizar un diagnóstico. Aunque hay muchas técnicas de análisis y diagnóstico corporativo, la microempresa solo necesita utilizar unos procesos simples. Acá se propone al cálculo basado en inductores de valor, los cuales están enfocados a medir la generación o destrucción de valor. Los inductores de valor, como su nombre lo dice, "inducen" a lograr mejores metas y objetivos e involucran a la empresa en la generación de valor (García, 2003, p.177).

Tomando como base los conceptos dados por García (2003) se seleccionan los inductores necesarios para apoyar este trabajo (ver Tabla 1), se toman los datos de los estados financieros proforma entre los años 2008-2013 que son obtenidos de la Superintendencia de Sociedades (Supersociedades, 2014). De estas se consideraron aquellas que cumplen con la condición de ser microempresas, para un total de un 169 empresas, de las que se tomó una al azar y se comparó su resultado con las otras del sector.

Tabla 1

Formulación y concepto sobre Inductores de Valor

\begin{tabular}{|c|c|}
\hline Tipo de inductor & Concepto \\
\hline $\begin{array}{l}\text { EBITDA = Utilidad Operativa + } \\
\text { depreciaciones y amortizaciones }\end{array}$ & $\begin{array}{l}\text { Muestra el resultado empresarial antes de } \\
\text { los intereses, impuestos, depreciaciones y } \\
\text { amortizaciones productivas. Resultado que } \\
\text { indica cuánto se convierte en efectivo y lo } \\
\text { que hay para el pago de impuestos, pasivos, } \\
\text { deuda con intereses y cuotas de amortización, } \\
\text { así como para invertir en el capital de trabajo, } \\
\text { para la reposición de activos y retribuir con } \\
\text { las utilidades al propietario. Su resultado } \\
\text { permite analizar el desempeño operativo de } \\
\text { una compañía (Jiménez, 2013). }\end{array}$ \\
\hline
\end{tabular}

Margen EBITDA = EBITDA / ventas Es una medida en sí de rentabilidad de un negocio, mide los centavos por cada peso como producto de ventas que se convierten en efectivo con el propósito de atender los compromisos más cercanos (Jiménez, 2013). 


\begin{tabular}{lrl}
\hline \multicolumn{2}{c}{ Tipo de inductor } & \multicolumn{1}{c}{ Concepto } \\
\hline KTNO (Capital de Trabajo & neto & Es importante que la empresa pueda contar \\
operativo $)=$ CXC + INV - CP & con liquidez, con el fin de atender todas las \\
& obligaciones, lo cual permite mejorar la liquidez, \\
& elemento clave del crecimiento. Para el cálculo \\
& KTNO (Capital de Trabajo neto operativo) se \\
& tiene en cuenta la suma de cuentas por cobrar \\
& (CXC), inventarios (INV) menos las cuentas por \\
& pagar (CXP) a proveedores de bienes y servicios \\
& $($ Jiménez, 2013).
\end{tabular}

EL INDUCTOR PKT (Productividad Refleja la eficacia de la administración en el del capital de trabajo $)=\mathrm{KTNO} /$ ventas uso de los recursos corrientes de la empresa (Jiménez, 2013).

PDC (Palanca de crecimiento) $=$ Calcula qué tan atractivo resulta para una Margen EBITDA / PKT empresa su crecimiento, desde el punto de vista del valor agregado. Si el resultado de la PDC es > 1, la empresa está lista para crecer; si es menor a uno (1), crecer deja de ser rentable, ya que el crecimiento compromete caja, lo que demanda más efectivo ocasionando un desbalance en el flujo de caja e impidiendo que se cumpla adecuadamente con los compromisos de pago a proveedores, de impuestos, servicio a la deuda, reposición de activos fijos y reparto de utilidades (Jiménez, 2013).

Elaboración propia con referencia en Jiménez, 2013.

Tabla 2

Otros indicadores de apoyo al análisis

El cálculo del indicador

Ciclo del efectivo $=$ Rotación de inventarios (días) + rotación de cuentas por cobrar (días) - rotación de las cuentas por pagar (días)

\section{Concepto}

Verifica la importancia de los inductores, se constata cómo los buenos resultados guardan una estrecha relación con ciclos de efectivo cortos, ya que se destina más capital a la reinversión, lo que mejora la rentabilidad y el endeudamiento. El ciclo de efectivo se calcula como el número de días que trascurren hasta que ingresa el efectivo producto de una venta, medido desde el momento en que se pagaron estos productos en el inventario (Jiménez, 2013). 


\begin{tabular}{ll}
\hline \multicolumn{1}{c}{ El cálculo del indicador } & \multicolumn{1}{c}{ Concepto } \\
\hline ROE = Utilidad Neta / Patrimonio Total & $\begin{array}{l}\text { Analiza la rentabilidad con el indicador de } \\
\text { retorno sobre el patrimonio (Jiménez, 2013). }\end{array}$ \\
\hline $\begin{array}{l}\text { ROI = (Ingresos - Costo de los } \\
\text { productos vendidos) / Costo de los } \\
\text { productos vendidos }\end{array}$ & $\begin{array}{l}\text { Mide el retorno sobre la inversión (Jiménez, } \\
\text { ROA = Utilidad Neta / Activo Total }\end{array}$ \\
& $\begin{array}{l}\text { Mide la rentabilidad del activo (Jiménez, } \\
2013) .\end{array}$ \\
\hline
\end{tabular}

Elaboración propia con referencia en Jiménez, 2013.

Son muchos los indicadores que se podrían aplicar como complemento de un diagnóstico, pero se observa que la formulación es simple porque puede ser aplicada por cualquier microempresario, incluso si se desea comparar los resultados de la empresa con otras del sector o analizar la gestión, solo se requieren tomar datos de los estados financieros clasificados tal como lo establece el Decreto 2649 y 2650 de 1993 (INCP, 2014).

\section{Resultados}

Realmente es poco lo que se ha profundizado en las pequeñas empresas sobre el tema de inductores de valor y mucho menos sobre su aplicación en la gestión de las Pymes. La mayoría de quienes administran microempresas no basan su gestión en el apoyo de este tipo de herramientas financieras (Jiménez, 2014). Pero veamos ¿qué es valor? La firma Stern Stewart \& Co, (2009) lo especifica como "una valoración de las ganancias obtenidas que difieren de la tasa de rentabilidad mínima exigida por los propietarios" lo que implica que es un indicador muy útil cuando se trata de evaluar el ejercicio y medir el logro de los objetivos (Jiménez, 2013).

Jiménez (2014) manifiesta que si los índices han ido avanzando, los empresarios deben apoyar su gestión con indicadores más dinámicos como son los llamados "inductores de valor", que conducen a la gerencia a lograr mejores resultados encaminándolos hacia creación de valor, porque el resultado permite analizar el desarrollo de los planes estratégicos. También comenta que los inductores de valor son una herramienta de control poco usada en la microempresa, resaltando lo importante y lo fácil que son de interpretar, porque ayudan a medir y a cuantificar datos, muestran diferencias de un periodo a otro y además permiten analizar las posibles causas y rediseñar las acciones en caso de problemas. 
La gerencia de una microempresa debe tener en cuenta los índices, los cuales son muy importantes de analizar los resultados, estos facilitan y ayudan a la gerencia a la planeación y permiten el control, los indicadores son de varios tipos, están aquellos por ejemplo que miden el crecimiento y la rentabilidad (Jiménez, 2014, p.52).

Por su parte, sobre el concepto de valor, el profesor García (2003) plantea que:

Se genera valor, cuando en las empresas los gerentes toman buenas decisiones, las cuales generan una rentabilidad superior al costo de capital, utilizado para financiar el ciclo operativo, si el rendimiento es menor, quiere decir que cada día más valor destruye y viceversa (p.27).

En 2010, Toro, en su percepción afirma que:

(...) los índices son instrumentos que inducen a equilibrar los resultados y son un soporte que sirve de plataforma para emprender tareas para los próximos periodos y para enfocar la empresa hacia la creación de valor, la trascendencia de estos indicadores, su continuo control y seguimiento son la base para tomar decisiones con criterio (pp.244-245).

Asimismo, García (2009) afirma que un inductor estima cómo se origina valor y calcula cuánto valor se crea o se destruye tomando el concepto desde la base de la rentabilidad. Los inductores de valor como indicadores son elementos esenciales que contribuyen con sus resultados a la generación de valor. García se cuestiona ¿cuántos gerentes efectúan cómputos antes de arrancar proyectos de desarrollo? La realidad, son escasos (Jiménez, 2013).

La importancia de los inductores con respecto a otros análisis tradicionales radica en que el cálculo pretende corregir las limitaciones de los indicadores clásicos, que no fundan su estudio en el significado del valor. La microempresa actual demanda de las gerencias asentarse en una plataforma con un punto de vista de creación de valor, la práctica de su uso se hace fundamental (Gómez, 2010).

En Colombia hay empresas con buenas ventas, generan utilidad, pero destruyen valor para su propietario. Esta investigación expone un modelo simple para ser aplicado a la microempresa, el resultado de calcular los inductores es observar cómo se afecta el valor de la empresa cada año, para tales efectos estos indicadores proporcionan datos que permiten apoyar la gestión con miras a administrar con éxito, plantear estrategias y enfocar la empresa a la generación de valor. 


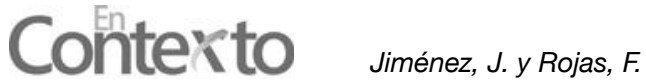

La información financiera se obtiene de los estados financieros de las microempresas del sector con código de clasificación CIIU-Rev. 3.1, G5131 que pertenece a la división de ventas al mayor de textiles, vestido y calzado (INEC, 2014). De la base de datos de la Superintendencia de Sociedades se seleccionan las microempresas que cumplen con esta condición, son 169 en total (Supersociedades, 2014), y se elige una al azar aplicando el método de número aleatorio; la empresa elegida fue Industrias Tago S. A. Para calcular los inductores tomamos los estados proforma de los años 2008-2013 y aplicamos las fórmulas para el cálculo según la Tabla 1.

Tabla 3

Cálculo del Inductor EBITDA

\begin{tabular}{lcccccc}
\hline Dato & 2008 & 2009 & 2010 & 2011 & 2012 & 2013 \\
\hline Utilidad operacional & 42 & -29 & 71 & 18 & 18 & 77 \\
\hline (+) Amortizaciones & 0 & 0 & 0 & 0 & 0 & 0 \\
\hline (+) Depreciaciones & 56 & 42 & 42 & 36 & 30 & 0 \\
\hline EBITDA (millones de pesos) & 98 & 13 & 113 & 54 & 48 & 77 \\
\hline
\end{tabular}

Elaboración propia basada en los estados financieros obtenidos de la Superintendencia de Sociedades años 2008-2013.

El Inductor EBITDA de Industrias Tago S. A. muestra que es capaz de generar efectivo y prueba que hay una utilidad operacional, pero el resultado final depende de la buena gestión administrativa, por esta razón este indicador no basta, y es necesario hacer un análisis más profundo. Ya se sabe que la empresa genera caja, pero este indicador debe analizase acompañado de otros antes de emitir una interpretación.

Tabla 4

Cálculo Inductor Margen EBITDA

\begin{tabular}{lcccccc}
\hline Dato (en millones de pesos) & 2008 & 2009 & 2010 & 2011 & 2012 & 2013 \\
\hline EBITDA & 98 & 13 & 113 & 54 & 48 & 76 \\
\hline Ventas & 1410 & 1286 & 1526 & 1685 & 1427 & 776 \\
\hline Margen EBITDA = EBITDA / Ventas & $7 \%$ & $1 \%$ & $7 \%$ & $3 \%$ & $3 \%$ & $10 \%$ \\
\hline
\end{tabular}

Elaboración propia basada en los estados financieros obtenidos de la Superintendencia de Sociedades años 2008-2013. 
El Margen EBITDAes un indicador de desempeño operacional que complementa al indicador EBITDA y permite ver la eficiencia de los ingresos por ventas generadas, el resultado expresa los centavos de cada peso producto de las ventas de Industrias Tago S. A., que se convierten en efectivo para atender los compromisos; para el año 2010 el 7\%, en el 2011 y 2012 bajó a un 3\% y el 2013 un $10 \%$ de cada peso se hacía efectivo.

Tabla 5

Cálculo Inductor KTNO (capital de trabajo neto operativo)

\begin{tabular}{lcccccc}
\hline DATO (en millones de pesos) & 2008 & 2009 & 2010 & 2011 & 2012 & 2013 \\
\hline CXC $=$ Clientes - Provisiones & 185 & 148 & 153 & 233 & 102 & 86 \\
\hline INV = Inventarios & 107 & 81 & 21 & 40 & 100 & 82 \\
\hline CXP = Cuentas por Pagar & 104 & 124 & 92 & 260 & 50 & 40 \\
\hline$K T N O=C X C+I N V-C X P$ & 188 & 105 & 82 & 13 & 152 & 128 \\
\hline
\end{tabular}

Elaboración propia basada en los estados financieros obtenidos de la Superintendencia de Sociedades años 2008-2013.

KTNO se puede expresar como el capital demandado por la empresa para tolerar el ciclo de efectivo requerido, lo ideal es que se pueda contar con la mayor liquidez posible que permita a la empresa cumplir con las obligaciones, lo que a la vez permite la generación de rentabilidad. La empresa en el 2012 requiere más capital, 152 millones de pesos, y baja para 2013 a 128 millones, lo cual hubiera sido positivo si se hubiera dado un incremento en ventas, pero esto no sucede. Como se verá este dato es fundamental para la gerencia y es fundamental para estimar las necesidades de capital en el periodo.

El concepto KTNO (capital de trabajo neto operativo) para Industrias Tago S. A. expresa la necesidad de conservar un ingreso firme de capital, y si el porcentaje de ahorro no le admite sostenerse entonces se tendrá que recurrir al crédito. La cuestión es ¿cuánto, cuándo y en qué momento endeudar la empresa? El cómputo del KTNO es una cifra muy importante para que el microempresario pueda saber cuánto efectivo se necesita para asegurar el funcionamiento. 


\section{Contexto unmeres, $u$ y prods, $F$}

Tabla 6

Cálculo Inductor productividad del capital de trabajo PKT

\begin{tabular}{lcccccc}
\hline Dato (cifras en millones de pesos) & 2008 & 2009 & 2010 & 2011 & 2012 & 2013 \\
\hline KTNO & 188 & 105 & 82 & 13 & 152 & 128 \\
\hline Ventas & 1410 & 1286 & 1526 & 1685 & 1427 & 776 \\
\hline Inductor PKT= (KTNO / VENTAS) & 0,13 & 0,08 & 0,05 & 0,01 & 0,11 & 0,16 \\
\hline
\end{tabular}

Elaboración propia basada en los estados financieros obtenidos de la Superintendencia de Sociedades años 2008-2013.

El inductor PKT (productividad del capital de trabajo) en Industrias Tago S. A. muestra cuántos centavos requiere la empresa para sostener un aumento de cada peso en ventas, el resultado guarda relación con el KTNO, pues cuanto más capital de trabajo se tenga que conservar por peso de ventas, mayor será la presión para el flujo de caja de la empresa y más cantidad de efectivo se necesitará para mantener el crecimiento. En el 2013 el indicador muestra un 0.16 , lo que señala una necesidad de efectivo y una destrucción de valor. Ahora bien, el incremento con respecto a los años anteriores se debe a las necesidades de KTNO, un manejo inadecuado trae efectos en la liquidez y el rendimiento del negocio. Se puede definir que el inductor Margen EBITDA expresa lo que deja la operación y la PKT refleja lo que consume la operación.

Tabla 7

Cálculo Inductor Palanca de crecimiento PDC

\begin{tabular}{lrrrrrr}
\hline Dato (millones de pesos) & 2008 & 2.009 & 2010 & 2011 & 2012 & 2013 \\
\hline Margen EBITDA & 0,07 & 0,01 & 0,07 & 0,03 & 0,03 & 0,10 \\
\hline PKT & 0,13 & 0,08 & 0,05 & 0,01 & 0,11 & 0,16 \\
\hline PDC & 0,52 & 0,12 & 1,38 & 4,15 & 0,32 & 0,60 \\
\hline
\end{tabular}

Elaboración propia basada en los estados financieros obtenidos de la Superintendencia de Sociedades años 2008-2013.

El cálculo del PDC surge de la relación entre el Margen EBITDA y PKT. El resultado muestra qué tanto es para la empresa crecer, es decir, si se generó valor. Por eso es importante que el resultado sea de una PDC mayor que 1, lo que supondría que el crecimiento libera caja, caso contrario sería si el indicador es menor a 1, lo que expresaría que el crecimiento deja de ser rentable (Jiménez, 2014). 
Para Industrias Tago S. A., el resultado para el 2013 es de 0.60, lo que muestra un crecimiento menor que uno (1), ello indica que la firma requiere más dinero para mantener la operación, en este caso no hay crecimiento, no se genera valor. En consecuencia Industrias Tago S. A. es menos rentable, así lo muestran los indicadores de ROE, ROA, ROI. La caída en ventas en Industrias Tago S. A. de los años 2011 y 2013 ocasiona un cambio abrupto de la PDC (palanca de crecimiento) pasando de cifras positivas a negativas. Esto quiere decir que la empresa demanda más efectivo para sostenerse en el 2012, produciendo un desbalance en el flujo de caja: una PDC inferior a uno (1) expresa que se puede llegar a dificultades para cumplir con el pago a los proveedores, al servicio a la deuda, a los impuestos y para los activos requeridos. Todo esto ocasionará que no se puedan distribuir utilidades. Por su parte, el reparto de utilidades, se observa cuando las necesidades de KTNO (capital de trabajo neto operativo) se incrementan de un periodo a otro, y las ventas disminuyen, por lo que se pueden originar problemas de liquidez.

Tabla 8

Cálculo Inductor el Ciclo del efectivo

\begin{tabular}{lcccccc}
\hline Dato & 2008 & 2009 & 2010 & 2011 & 2012 & 2013 \\
\hline Días de cxc = 365 / (ventas / clientes) & 48 & 42 & 37 & 50 & 26 & 40 \\
\hline Días de inventario = 365 / (cmv / inv.) & 32 & 25 & 6 & 10 & 28 & 43 \\
\hline Días de prov. = 365 (cmv / prov.) & 31 & 38 & 26 & 62 & 14 & 21 \\
\hline Ciclo del efectivo en días & 49 & 29 & 17 & -2 & 40 & 62 \\
\hline
\end{tabular}

Elaboración propia basada en los estados financieros obtenidos de la Superintendencia de Sociedades años 2008-2013, Abreviaturas: CXC = Cuentas por Cobrar; CMV = Costo de la mercancía vendida; Prov.: proveedores inv. = Inventarios. Cifras en millones de pesos.

El ciclo de efectivo corrobora todo lo anteriormente expuesto. Cuánto más largo sea el ciclo de efectivo, más efectivo se necesita para sostener la operación, y ese es precisamente el caso de Industrias Tago S. A. El ciclo del año 2012 requirió 40 días y en 201362 días, siendo éste el tiempo que tarda un peso invertido en volver a la empresa. Esta mayor necesidad de fondos, producto de la caída en ventas, provoca justamente el incremento del ciclo de efectivo, el cual ve reflejado en una mayor necesidad de KTNO (capital de trabajo neto operativo), ver Tabla 5. 


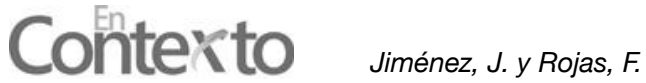

Lo ideal para una empresa que obtiene liquidez, es reinvertir, lo que evita el endeudamiento y el costo de intereses. Los microempresarios deberán saber calcular debidamente las necesidades de efectivo, porque un manejo inadecuado del ciclo de efectivo puede llevar a una quiebra rápidamente o a la insolvencia (Arronte, 2008, p.19). Igualmente, deben calcular muy bien las necesidades de efectivo requeridas, pues un cálculo inadecuado puede afectar las utilidades y limitar el funcionamiento de la empresa (Gitman, 2003, p.486).

La rentabilidad depende, entonces, de un manejo adecuado del KTNO, este varía en función inversa a la liquidez, por eso no se debe dejar el dinero en caja más del tiempo necesario. El dinero debe rotar lo más rápido posible, con el fin de reconvertir lo más pronto, esto es lo que origina el crecimiento, y, solamente lo obtendrán las empresas que manejen el ciclo de efectivo con más eficiencia. "El objetivo de un diagnóstico es la de evaluar los factores económicos que determinan la rentabilidad de las empresas, se busca interpretar los factores que permitan la permanencia en el medio" (Horne y Wachowicz, 2002, pp.208250). Lo ideal es contar con ciclos de efectivo cortos, lo cual se relaciona con una mayor rentabilidad (Mora y Benavides-Franco, 2008, p.2).

Tabla 9

Cálculo Indicador de Rentabilidad ROE, ROA, ROE

\begin{tabular}{lcccccc}
\hline Datos & 2008 & 2009 & 2010 & 2011 & 2012 & 2013 \\
\hline Retorno / patrimonio (ROE) & nd & $-15,00 \%$ & $6,00 \%$ & $4,00 \%$ & $3,00 \%$ & $0,45 \%$ \\
\hline Retorno sobre la inversión (ROI) & $3,26 \%$ & $-8,00 \%$ & $2,00 \%$ & $1,10 \%$ & $0,72 \%$ & $0,36 \%$ \\
\hline Retorno sobre los activos (ROA) & $11,00 \%$ & $-7,00 \%$ & $3,00 \%$ & $2,00 \%$ & $0,73 \%$ & $0,36 \%$ \\
\hline
\end{tabular}

Elaboración propia basada en los estados financieros obtenidos de la Superintendencia de Sociedades años 2008-2013.

Los indicadores de rentabilidad de la empresa Industrias Tago S. A. no muestran los mejores resultados. El ROA, ROE, ROÍ cada año son más bajos, no llegan para el 2013 ni siquiera al uno por ciento, y la caída en ventas y el resultado poco favorable de los indicadores, son el reflejo de cómo se afecta la gestión administrativa, ver Tabla 9. 
Tabla 10

Análisis inductores Industrias Tago S. A. Y su comportamiento frente a la dinámica del Sector años 2011-2013

\begin{tabular}{lcccccc}
\hline \multirow{2}{*}{ Resumen } & \multicolumn{3}{c}{ Industrias Tago S.A. } & \multicolumn{3}{c}{$\begin{array}{c}\text { Sector. Cálculo promedio a } \\
169 \text { empresas del sector }\end{array}$} \\
\hline Cálculos & 2011 & 2012 & 2013 & 2011 & 2012 & 2013 \\
\hline Ventas & 1685 & 1427 & 776 & 34.612 & 13.738 & 15.436 \\
\hline Crecimiento ventas & $10,42 \%$ & $-15,31 \%$ & $-45,60 \%$ & $179 \%$ & $-60 \%$ & $12 \%$ \\
\hline KTNO & 13 & 152 & 128 & 18.726 & 5.802 & 5.892 \\
\hline EBITDA & 54 & 48 & 77 & 3.698 & 898 & 1.183 \\
\hline Margen EBITDA & 0,03 & 0,03 & 0,1 & 0,11 & 0,07 & 0,08 \\
\hline PKT & 0,01 & 0,11 & 0,16 & 0,54 & 0,42 & 0,38 \\
\hline PDC & 4,15 & 0,32 & 0,6 & 0,2 & 0,15 & 0,2 \\
\hline Ciclo de efectivo / días & $-2,39$ & 40,3 & 62,1 & 48,777 & 28,66 & 16,843 \\
\hline ROE & $4,00 \%$ & $3,00 \%$ & $0,45 \%$ & $12,81 \%$ & $11,42 \%$ & $7,18 \%$ \\
\hline ROÍ & $1,10 \%$ & $0,72 \%$ & $0,36 \%$ & $3,80 \%$ & $4,14 \%$ & $2,87 \%$ \\
\hline ROA & $2,00 \%$ & $0,73 \%$ & $0,36 \%$ & $2,55 \%$ & $2,78 \%$ & $1,92 \%$ \\
\hline
\end{tabular}

Elaboración propia basada en los estados financieros de 169 empresas del sector comercio clasificación CIIU-Rev. 3.1, G5131, tomados de la Superintendencia de Sociedades 2011-2013. Cifras en millones de pesos.

Es lógico pensar que el desempeño empresarial se vea afectado por asuntos de la economía o por problemas generalizados en el sector de la economía en el que se bate la empresa. Se espera que si las ventas caen, la situación sea igual para todas las empresas, pero no es así, por eso se propone comparar la situación de Industrias Tago S. A. con el resto del sector micro empresarial. Para tales efectos, se realizó un idéntico cálculo a los estados financieros de las 169 empresas del sector, determinando así los promedios por los años 20112013, ver Tabla 10.

Al observar las ventas se puede señalar una caída para el año 2013 en Industrias Tago S. A. de un $-45,60 \%$, pero no así para el sector compuesto por 169 empresas del sector micro empresarial que logra incrementar las ventas en un $12 \%$. La rentabilidad del sector en promedio es mucho mejor, $7,18 \%$ en el indicador ROE, que es un indicador muy utilizado para la comparación de la rentabilidad de empresas del mismo sector. Si se compara con el resultado obtenido por Industrias Tago S. A., en el 2013 solo tiene el 0.45\%, ni siquiera 
el 1\%. El mismo comportamiento presenta el indicador de rentabilidad ROE, ROÍ y ROA; ver Tabla 10.

Si se sigue la premisa de que ciclos cortos arrojan mejores rentabilidades, se peude afirmar que, en efecto, esa precisa se aplica explicar la pérdida de rentabilidad de Industrias Tago S.A, porque se observa cómo el promedio del sector micro empresarial maneja un ciclo de efectivo más corto para el año 2013, tan solo de 16 días, en comparación con el ciclo de efectivo de 62 días de Industrias Tago S. A..

Se espera que los requerimientos de capital de trabajo neto operativo (KTNO) se muevan en igual proporción a las ventas, lo que no sucede para Industrias Tago S. A., pues las ventas caen en 2012 un $15 \%$ y en 2013 un 45.6\%, pero no los requerimientos de KTNO, ya que en el 2012 se requirió \$152 millones de pesos y en el 2013 bajó a $\$ 128$ millones, variando solamente un 12.5\%. Esto muestra que se cubre la operación con efectivo de periodos anteriores, ver Tabla 10. El resultado EBITDA y el Margen EBITDA indican que los costos de producción son altos y dejan un margen operativo en porcentaje inferior para el sector e Industrias Tago S. A.. Frente al sector en general, la empresa tiene costos más altos, lo que no permite contribuir con una mejor rentabilidad, el efectivo que se obtiene apenas cubre las necesidades de la empresa.

La productividad del capital de trabajo PKT, tanto para el sector como para Industrias Tago S.A., no es favorable, y la relación, en cuanto a los indicadores Margen EBITDA y PKT, se deben controlar continuamente. El primero no debe disminuir ni mucho menos ser inferior al segundo, ya que no se genera valor, cuando el Margen EBITDA es mayor que la PKT, se da el crecimiento, ya que se libera caja. La relación entre los anteriores indicadores debe ser mayor que 1 , si se da un resultado inferior, quiere decir que para la empresa no es interesante crecer.

Realizado el diagnóstico a Industrias Tago S. A. se observa que el dinamismo de la empresa no es el mejor, con una rentabilidad inferior al 1\%, pero tampoco el sector marcha a pleno, su mejor cifra es un ROE del 7.18\%. Para este tipo de empresas los resultados económicos no han sido lo mejor, su rentabilidad debería estar por encima de estos resultados, pero no es así, basta señalar que el margen de la utilidad de las empresas colombianas en promedio es de $14,5 \%$ (Periódico Portafolio, 2015).

Un modelo claro para Colombia fue la publicación de la Revista Dinero, donde se reseña una investigación de la firma Stern Stewart \& Co. (2009), según la cual el $23 \%$ de las grandes empresas lograron generar valor durante el año 
2009 , teniendo rendimientos sobre el capital invertido entre el $13 \%$ y el $35 \%$, mientras que el $77 \%$ de las grandes empresas destruyeron valor, de las cuales el 16\% tuvo rentabilidades negativas en su rendimiento de capital. Si este es el resultado para las grandes empresas se puede analizar que para la microempresa es aún más difícil generar valor (Revista Dinero, 2010).

Todo esto quiere decir que las medianas y pequeñas empresas se sostienen, no crecen y no generan valor para sus propietarios. El análisis de los inductores vistos se debe entender como una herramienta base para el control, con miras a estudiar sus resultados para tener un mayor resultado operativo.

\section{Conclusiones}

Los inductores de valor son importantes al comparar una microempresa con otras del sector, así como para medir la tendencia del negocio. Por eso es tan importante que los empresarios comprendan la necesidad de administrar con base a planes y metas. Estos indicadores son un instrumento de apoyo para la toma de decisiones fundamentales como fuente de información en el diseño de estrategias, además son fáciles de calcular y orientan la empresa hacia la creación de valor. Con este sencillo modelo de diagnóstico se pudo apreciar que es posible aplicar análisis simples en las Pymes que permitan obtener los resultados de una empresa y compararlos frente a la dinámica del sector.

Se apreció en el diagnóstico lo fácil que es aplicar inductores generadores de valor, basta con unos pocos indicadores para obtener información clave. Manejar bien el capital es fundamental y cualquier problema al interior de la empresa afecta las finanzas. La realidad es que muchos empresarios administran basándose en utilidades y no en la rentabilidad, sin planeación y sin estrategias, lo que de por sí afecta el crecimiento, pués si un peso se tarda demasiado en volver a la empresa, entonces aquello que se gana o bien se gasta o se consume inmediatamente, es decir, no se crea valor.

Por esto, la rentabilidad debe ser el objetivo económico en toda organización, es lo que esperan los propietarios y es uno de los conceptos menos comprendidos, por eso es necesario que la dirección plantee sus estrategias orientadas a la búsqueda de resultados financieros. La gerencia debe empezar a plantear metas y perseguir el logro de objetivos, basados en el cálculo y seguimiento de indicadores, por ejemplo mejorar el EBITDA, aumentar la productividad y un crecimiento basado en el indicador PDC mayor a uno. La administración basada en el valor es una estrategia gerencial que puede llevar a la compañía a otro nivel de competencia. 


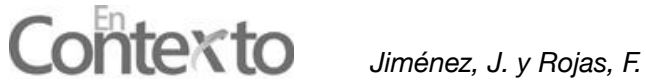

Se observa cómo los resultados para el sector e industrias Tago S. A. no son los mejores, la economía no marcha bien. Y siendo más comprometedores los resultados para la empresa se aprecia baja rentabilidad, no se genera valor y sus indicadores están muy por debajo del sector, tampoco se logra un buen desempeño, generando que la empresa comprometa excesivos recursos en mantener inventario, cuentas y documentos por cobrar. Todo esto refleja ineficiencias administrativas, por eso el resultado del inductor PKT no es el más óptimo, lo que a su vez causa incremento de las necesidades de capital trabajo neto que, a su vez, retraduce en un ineficiente ciclo de efectivo.

La palanca de crecimiento PDC para Industrias Tago S. A. muestra que no hay crecimiento, no se genera valor, pues el resultado obtenido es inferior a uno, y en lugar de redimir efectivo se gasta el de ciclos anteriores, lo que provoca un desequilibrio en el presupuesto, afectando las necesidades de caja. Por eso se requiere plantear estrategias con el apoyo de indicadores para establecer metas de crecimiento basados en perseguir mejores resultados, por ejemplo con el indicador PDC buscar que sea mayor a uno también es necesario llevar la organización a otro nivel de competencia, por eso es un punto álgido la PKT (productividad del capital de trabajo): se destinan exagerados recursos a inventarios o en documentos y cuentas por cobrar lo que obliga a las gerencia a maniobrar con baja productividad, menoscabando la renta de la compañía y colocándola en riesgo por escaza liquidez.

Algo positivo del tema sobre inductores de valor para los empresarios está en que estos pueden establecer los inductores que adquieren predominio y cuáles variables perturban el dinamismo de la industria, o sea, cuáles aquejan concisamente la creación o destrucción del valor. Los gerentes Pyme modernos deben estar a la delantera en el uso de métodos y herramientas gerenciales que les faciliten elementos técnicos para el análisis y control de las operaciones, y así puedan contrastar los resultados que le son adversos y los conduzcan a la búsqueda de valor; los inductores ayudan a obtener mejores resultados en la inversión.

\section{Referencias}

Arronte, R. (2008). Metodología para proyectar una empresa industrial en México. Ciudad de México, México: Universidad Nacional Autónoma de México. 
Benjumea, B. (1 de marzo de 2012). Las Pymes también necesitan gerentes. Periódico el Colombiano. Recuperado de http://www.elcolombiano. com/BancoConocimiento/las_pymes_tambien_necesitan_gerentes/las_ pymes_tambien_necesitan_gerentes.asp.

Decreto 2649 (diciembre 29 de 1993). Por el cual se reglamenta la Contabilidad en General y se expiden los principios o normas de contabilidad generalmente aceptados en Colombia. Diario Oficial, 41.156, 1993, 29, 12. Recuperado de http://www.alcaldiabogota.gov.co/sisjur/normas/ Norma1.jsp?i=9863

El EVA de las 5000 (28 de mayo de 2010). Revista Dinero. Recuperado de http:// www.dinero.com/edicion-impresa/caratula/articulo/el-eva-5000/96285.

García, O. (2009). Sistema de creación de valor. Cali, Colombia: Prensa Moderna Impresores S.A.

García, O. (2003). Valoración de Empresas, Gerencia del Valor y EVA. Cali, Colombia: Prensa Moderna Impresores S.A.

Gitman, L. (2003). Administración de capital de trabajo y de activos circulantes. Ciudad de México, México: Editorial Pearson.

Gómez, J. (2010). Cartilla financiera. Bogotá, Colombia: Universidad Nacional de Colombia.

Horne, J. y Wachowicz, J. (2002). Fundamentos de administración financiera. Ciudad de México, México: Pearson Educación.

Jiménez, J. (2013). El inductor-PDC enfocado a medir el crecimiento y generación de valor para la PYME. Dimensión Empresarial, 13(2), 151-165.

Jiménez, J. (2014). La gerencia en el manejo del efectivo y los efectos frente al endeudamiento y rentabilidad. I Congreso Internacional de Finanzas, Universidad Pontificia Bolivariana, Bucaramanga. Recuperado de http:// congresofinanzas.upbbga.edu.co/2014/public/document/e-book_I_ Congreso_Internacional_de_Finanzas.pdf

Ley 550. (1999). Por la cual se establece un régimen que promueva y facilite la reactivación empresarial y la reestructuración de los entes territoriales para asegurar la función social de las empresas y lograr el desarrollo armónico de las regiones y se dictan disposiciones para armonizar el régimen legal vigente con las normas de esta ley. Recuperado de http:// www.secretariasenado.gov.co/senado/basedoc/ley_0550_1999.html

Mora, M. y Benavides, J. (2008). Efecto del ciclo de efectivo sobre la rentabilidad de las firmas colombianas. Cuadernos de Administración 21(36) 167-182. 


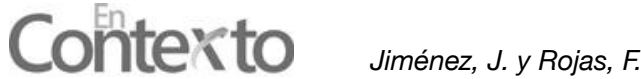

Ospina, O. (2013). Causas de MORTALIDAD DE LAS MICRO Y PEQUEÑAS EMPRESAS EN ANTIOQUIA. Revista Antioqueña de Economía y Desarrollo, 7, 6-49. Recuperado de ttp://www.camaramedellin.com. co/site/Portals/0/Documentos/2013/Revista_Antioque\%C3\%B1a_ Economia_Desarrollo7.pdf?utm_source=RAED6_home\&utm medium $=$ web\&utm_campaign=RAED6

Periódico Portafolio. (28 de 02 de 2015). Rentabilidad de las empresas. Recuperado de http://www.portafolio.co/negocios/rentabilidad empresas.

Santos, D. (1998). Trampas al crecimiento. Madrid, España: Ediciones Díaz de Santos.

Stern Stewart \& Co. (2012). Consultoría en administración y consultoría basada en el valor, EVA. Recuperado de http://www.ucema.edu.ar/ conferencias/download/2012/04.24F2.pdf

SIREM. (2016). Sistema de información y reporte empresarial Superintendencia de Sociedades. Recuperado de http://sirem.supersociedades.gov.co/ Sirem2/index.jsp

Toro, D. (2010). Creación de valor financiero. Bogotá, Colombia: ECOE EDICIONES.

\section{Para citar este artículo:}

Jiménez, J. y Rojas, F. (2016). Aplicación de inductores generadores de valor para la gestión en microempresas. En-Contexto, 4(5), 99-116. 